\title{
Active Versus Passive Flow Control in UVC FILTERs for COVID-19 Containment
}

\author{
E. Moreno ${ }^{1},{ }^{1}$ G. KlochoK, ${ }^{2}$ and S. García ${ }^{3}$ \\ ${ }^{1}$ Univ Lyon, UJM-Saint-Etienne, CNRS, Institute of Optics Graduate School, Laboratoire Hubert Curien UMR5516, 42023 St- \\ Etienne, France; ${ }^{2}$ Moscow State University of Civil Engineering, National Research University, Yaroslavskoye Shosse, Moscow, \\ Russia; and ${ }^{3}$ EULEN Group, Neurotraumatology and Rehabilitation Hospital, Granada, Spain
}

(Received 7 April 2021; accepted 16 June 2021; published online 30 June 2021)

Associate Editor Stefan M Duma oversaw the review of this article.

\begin{abstract}
Ultraviolet radiation as a germicide is widely used in the health field and even in domestic hygiene. Here, we propose an improvement in low-cost portable units of filtration for indoor air, which is based on ultraviolet radiation. In the current technology, to carry out an air filtration with a suspension of aerosols in which there is a likely concentration of pathogens, whether viral, bacterial or molds, the air is forced to pass as close as possible to the ionizing radiation source (near field). Since the optical mass is very small, the desired effect can be achieved in a considerably short time, deactivating the infective potential of these biological agents. The proposal of this work is the regulation of the flow or speed control of passage through these filters by passive elements instead of by electronic control systems. For this, two devices have been designed, simulated, and built, obtaining similar net pathogen inactivation rates under different flow rates. The passive flow control device has demonstrated higher performance in terms of flow rate and lower cost of production since they do not require electronics and are produced with fewer diodes. This passive device has also shown a lower projection of maintenance cost, lower energy consumption rate (higher efficiency), and longer projection of useful life.
\end{abstract}

Keywords-Indoor air purification, Passive flow control, Pathogen inactivation, COMSOL Flow Algebraic yPlus interface, COVID-19, SARS-CoV-2, UVC, Photo diodes.

Address correspondence to E. Moreno, Univ Lyon, UJM-SaintEtienne, CNRS, Institute of Optics Graduate School, Laboratoire Hubert Curien UMR5516, 42023 St-Etienne, France. Electronic mail: enrique@moreno.ws

\section{INTRODUCTION}

The COVID-19 interstitial pneumonia is an abrupt progression to respiratory failure ${ }^{6}$ which is associated with some serious coagulant complications. ${ }^{3}$ In ${ }^{9}$ the authors pointing out that this abrupt deterioration may be caused by a sudden shift in the spread of virusladen bioaerosols through the airways to many different regions of the lungs from the initial site of infection. In particular, the bioaerosols are more dangerous in close spaces, indoor spaces are the prime COVID hotspots. ${ }^{13}$ This article aims to contribute to the improvement of air quality, not only thinking about Covid-19 but also about all kinds of pathogens that are transmitted by aerosols. In ${ }^{15}$ the authors suggest that of the existing devices, all have shortcomings which render them ineffective and impractical in out-of-hospital environments. So as to overcome this lack, we deal with the problem by proposing wide spectra applicability devices that are cheap, simple use, low consumption, and mechanically compact.

In ${ }^{4}$ a group of individuals in the Biomedical Engineering (BME) community have created a document to help collect and disseminate guidance for those who teach in BME to move lectures, labs, and design projects online. The goal of this was to facilitate a community-wide virtual brainstorm on the topic. During the pandemic year 2020, there have been many similar initiatives to combat the disease. The origin of this work can be found in one of them, the OpenBridge Covid-19 - Santander X, where the first author and two students at the University of Chile participate with a rudimental first idea based on ultraviolet light. According to the World Health Organization, there 
exists three kinds of ultraviolet (UV) radiation ${ }^{18}$ which are classified attending to their wavelength in UVA (315-400 nm), UVB (280-315 nm), and UVC (100-280 $\mathrm{nm})$. The latter type, the UVC radiation, shows a high ionization capacity of the chemical bond, and this makes it especially interesting as a sterilizer, and it is considered in many devices for this purpose. ${ }^{8-20}$ In all technologies, there is a common link; the air to be filtered is made to pass close to the source of UVC radiation, forcing the flow through mechanical means (pumps or fans). In these devices, it is assumed that the radiative level is so intense that in fractions of a second, the pathogens traveling on aerosols are neutralized. Versus the mercury lamps, which are more powerful, we have employed solid-state diodes in our scheme. Both Hg-lamps and diodes can emit in UVC, but in front of the powerful lamps, the diodes are able to generate higher densities of irradiation locally. Therefore, some provisions must be made for its use to be effective. The most important is to control the exposure time of the biological agents carried by the aerosols (viruses, bacteria, fungi, protozoa, ...). When using devices that have intermediate radiative power, it is necessary to increase the exposure time so that the ionization effect takes place mainly at the optical mass considered. To the question of why use diodes instead of lamps, the answer has three considerations. Firstly, they consume less energy. Secondly, they are safer due to the local character of their emission, and thirdly they are more compact, allowing this to develop smaller devices. ${ }^{22}$ Although mercury lamps have a higher fluence, when we consider the energy consumed, if we match that energy with an equivalent number of diodes, we obtain a greater radiative capacity. Furthermore, diodes can be spatially distributed as concentrated sources of radiation. Let us not forget that the intensity of an electromagnetic source falls quadratically with distance. Thus, in devices such as ${ }^{14}$ and ${ }^{20}$, we find flow points very far from the source. When we say very far away, we are considering points that are far from the source millions of times the wavelength associated with UVC radiation.

In this work, in order to compare, we have designed, simulated, and built two different devices. These aim to regulate the air flux to set the exposure time to UVC radiation at values according to the light power supplied by the LEDs. We begin by describing the active flow control device or device one. In this device, an electronic microcontroller (ARDUINO UNO), which is illustrated in Figs. 1 and 3(b), controls the flow rate. As can be seen in Fig. 1, the geometry of the filter chamber is very similar to a funnel. In such a way that the air is forced to circulate inside a cylindrical column in which six rings with three LEDs each have been

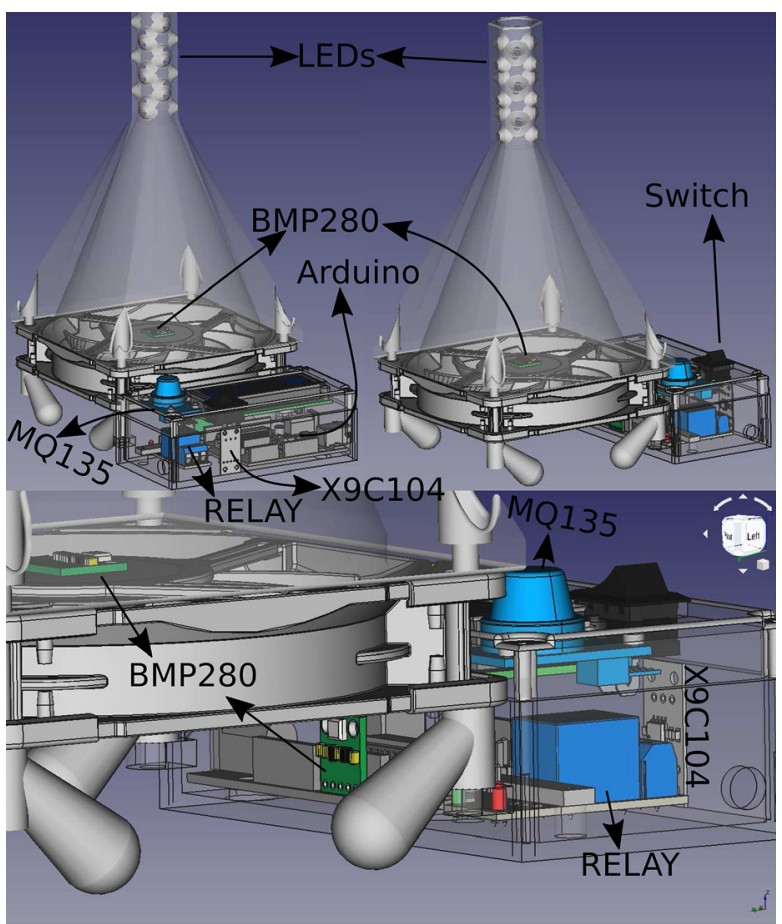

FIGURE 1. Schematic of the electronically controlled device. The figure shows the control components together with the structure through which the air flows to be filtered.

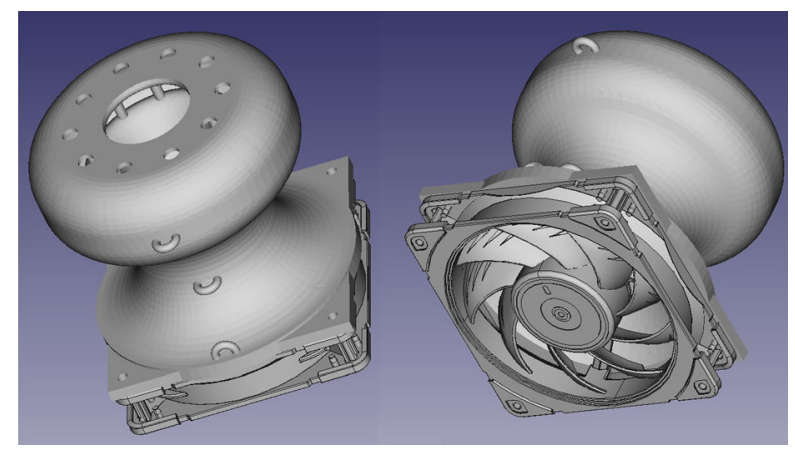

FIGURE 2. Illustration of the device that regulates the air flow passively. In this device, the geometry is responsible for regulating or controlling the speed of the fluid.

arranged. Two rings superimposed in plan form a regular hexagon since the

arrangement of the LEDs in each ring is 120 degrees. The vertical distance that exists between each ring is such that the non-existence of non-illuminated points in the column is ensured. This implicates accepting the Stanley specifications. ${ }^{19}$ In these specifications, the maximum radiance or directivity provided by the manufacturer is 60 degrees (see figure corresponding to directivity on the manufacturer's sheet. ${ }^{19}$ ).

In device one the flow rate is set by the fan's working regime, which is electronically controlled. In the second device, shown in Figs. 2 and 3a), it is the 
dynamics of the fluid/air, itself in its interaction with the walls of the filter chamber, the agent that regulates the flux. And this phenomenon is quite independent of the working regime of the fan.

It is intuitive to envision that a device with fewer moving parts and more simplicity of operation and construction will have greater advantages. Especially if it presents similar features (pathogen deactivation capabilities). It is at this point that we are going to demonstrate that a particular geometry can help to perform the same function, with higher performance, with the advantages that this entails.

In addition to this introduction, the rest of the manuscript is divided into three more sections. In section 2 we introduce the electronics that control the active filter. In section 3, we present the simulations carried out and based on the genetic degradation properties of certain pathogens, we discuss the devices' capabilities that have been considered in this study.

\section{CONTROL CIRCUIT}

The electronically controlled flow device has an ARDUINO UNO microcontroller to manage it. ${ }^{23}$ The microcontroller operates based on various sensors. Thus, an MQ135 sensor measures the concentration of carbon dioxide (proportional to the number of people in a room and the time they have been there without ventilating that room). This sensor is the one that activates both the fan and the LEDs through a relay. This would be the smart mode of operation of the device. But we can also override the decisions made by the microcontroller through the carbon dioxide concentration values provided by the sensor. In other words, we can make the system filter continuously. In that case, both the UVC LEDs and the fan run all the time. Establishing in this form a continuous mode of operation. For this purpose there is a switch that can be seen in both Figs. 3(b) and 4. The latter schematizes the circuit diagram. In addition to this, to reduce the flow rate in the radiation column, the pressure difference between the outside of the filter and the filtering chamber is measured. For this, and as illustrated in Fig. 4, two pressure probes BMP280 are placed that provide the difference. With this data, the microcontroller is able, through the use of a digital potentiometer X9C104 combined with a transistor BC335, to regulate the

fan speed so that the fan can be operated from $17 \%$ to $100 \%$ of its capacity or speed of rotation. In most tests the fan will operate around $30 \%$ to maintain a flow rate of $0.084 \frac{L}{s}$ or $0.3 \frac{m^{3}}{h}$. Later we will discuss the details related to the radiation dose that a pathogen would receive in this operating regime and that leads

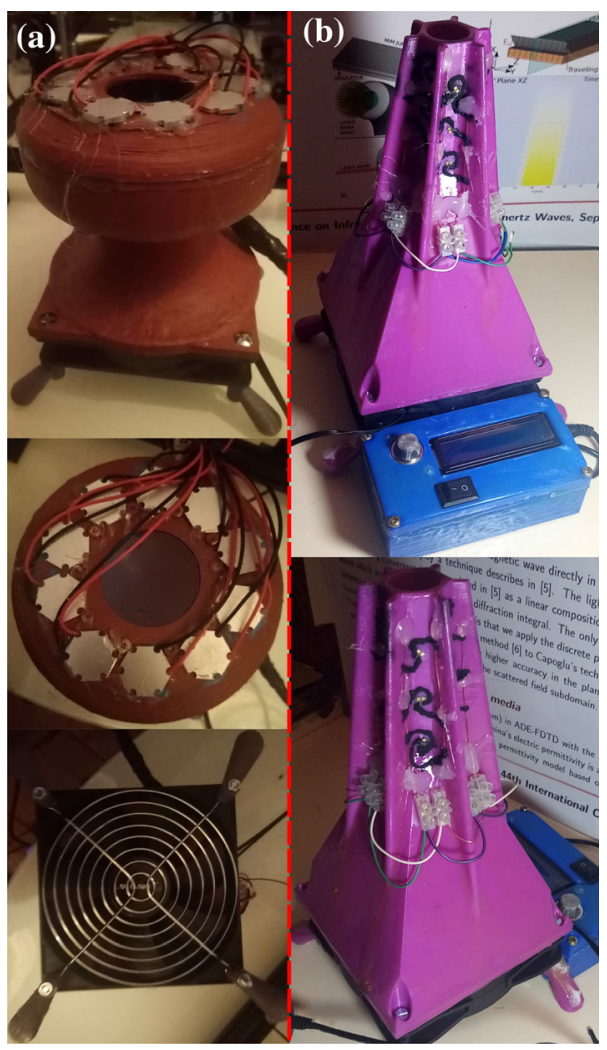

FIGURE 3. (a) Photo of device two, (b) photo of device one.

us to affirm that $99 \%$ of the pathogens would lose their infective capacity. Obviously, the ability to filter or deactivate the infectivity of potential pathogens can be sacrificed by virtue of a larger filtered volume by increasing the flow rate.

\section{SIMULATIONS AND RESULTS}

We have divided the simulations into two blocks. In the first block, we treat the fluid dynamics, and in the second block, the electromagnetic radiation present in the filter column.

\section{Air flow}

In addition to measuring the flow rate provided by this first device, the flow dynamics were also simulated assuming a turbulent regime. Thus, the density of velocity distributions inside the filter chamber and the pressures were determined. The simulations used the commercial software package COMSOL 5.0. ${ }^{2} \mathrm{We}$ employ the Turbulent Flow Algebraic yPlus interface. It is used for simulating single-phase flows at high Reynolds numbers. Although the Reynolds number could indicate that we are in a laminar regime, the strong vorticity presented by the device shown in 


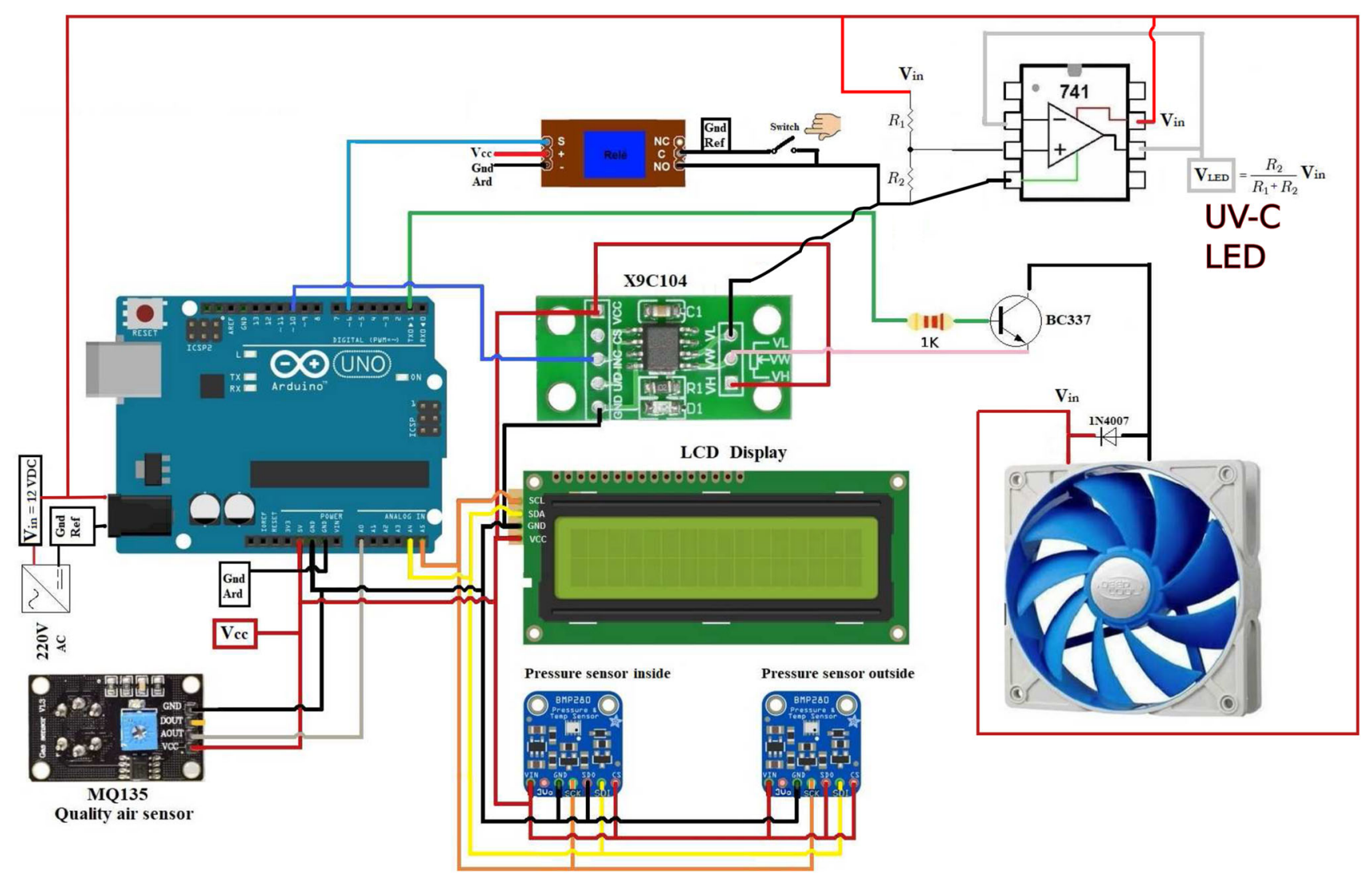

FIGURE 4. Control circuit for device one. The core of this circuit is an open-source Arduino UNO microcontroller.

Fig.5b) and the effect of passing through the cavities where the diodes are located in the device shown in the Fig.5a) justifies the choice of a calculation or modeling under the assumption of turbulent regime. The physics interface is suitable for incompressible flows and compressible flows at low Mach number (typically less than 0.3). The equations solved by the Turbulent Flow Algebraic yPlus interface are the Reynolds averaged Navier-Stokes equations for conservation of momentum and the continuity equation for conservation of mass. Turbulence effects are included using an enhanced viscosity model based on the local wall distance. Figs. 5(a) and (b) depict the computational domain under consideration for both filters.

The physics interface therefore includes a wall distance equation. We solve stationary regime.

In summary it was resolved the following coupled partial differential equations ${ }^{5,25}$ :

$$
\begin{gathered}
\rho(\mathbf{u} \cdot \nabla) \mathbf{u}=\nabla \cdot\left[-P \mathbf{I}+\left(\mu+\mu_{T}\right)\left(\nabla \cdot \mathbf{u}+(\nabla \cdot \mathbf{u})^{T}\right)\right]+\mathbf{F} \\
\rho \nabla \cdot \mathbf{u}=0
\end{gathered}
$$

$$
\left(1+2 \sigma_{w}\right) G^{4}=\nabla G \cdot \nabla G+\sigma_{w} G(\nabla \cdot \nabla G)
$$

Where $\mathbf{u}$ and $P$ are the field of velocities and the pressure, respectively, the earth's gravitational field is introduced by means of the force per unit of mass of fluid $\mathbf{F}$ and $\mathbf{I}$ is the unit tensor. In the continuity equation $\frac{\partial \rho}{\partial t}+\nabla \cdot(\rho \mathbf{u})=0$, which represents the conservation of mass, we assume a constant density for the air $\frac{\partial \rho}{\partial t}=0$. In few words, we have small changes in the pressure and then in the density, so we assume an incompressible fluid where the density is constant. Consistent with the steady regime assumed there is no change in the field velocity along the time, so in the vector equation which represents conservation of momentum $\rho \frac{\partial \mathbf{u}}{\partial t}+\rho(\mathbf{u} \cdot \nabla) \mathbf{u}=\nabla \cdot \sigma+\mathbf{F}$ the term $\rho \frac{\partial \mathbf{u}}{\partial t}$ has been omitted under the consideration of its null value. Obviously, in these equations $\sigma=-P \mathbf{I}+\epsilon$ is the total stress tensor defined as a sum where the viscous stress tensor $\epsilon$ is defined through the strain rate tensor $\nabla \cdot \mathbf{u}+(\nabla \cdot \mathbf{u})^{T}$ where we assume a general Newtonian media. In this context, $\mu$ is the dynamics viscosity, $\rho$ is the air density, and $G$ the reciprocal wall distance. This last term needs an explanation. Turbulence models often use the distance to the closest wall to approxi- 


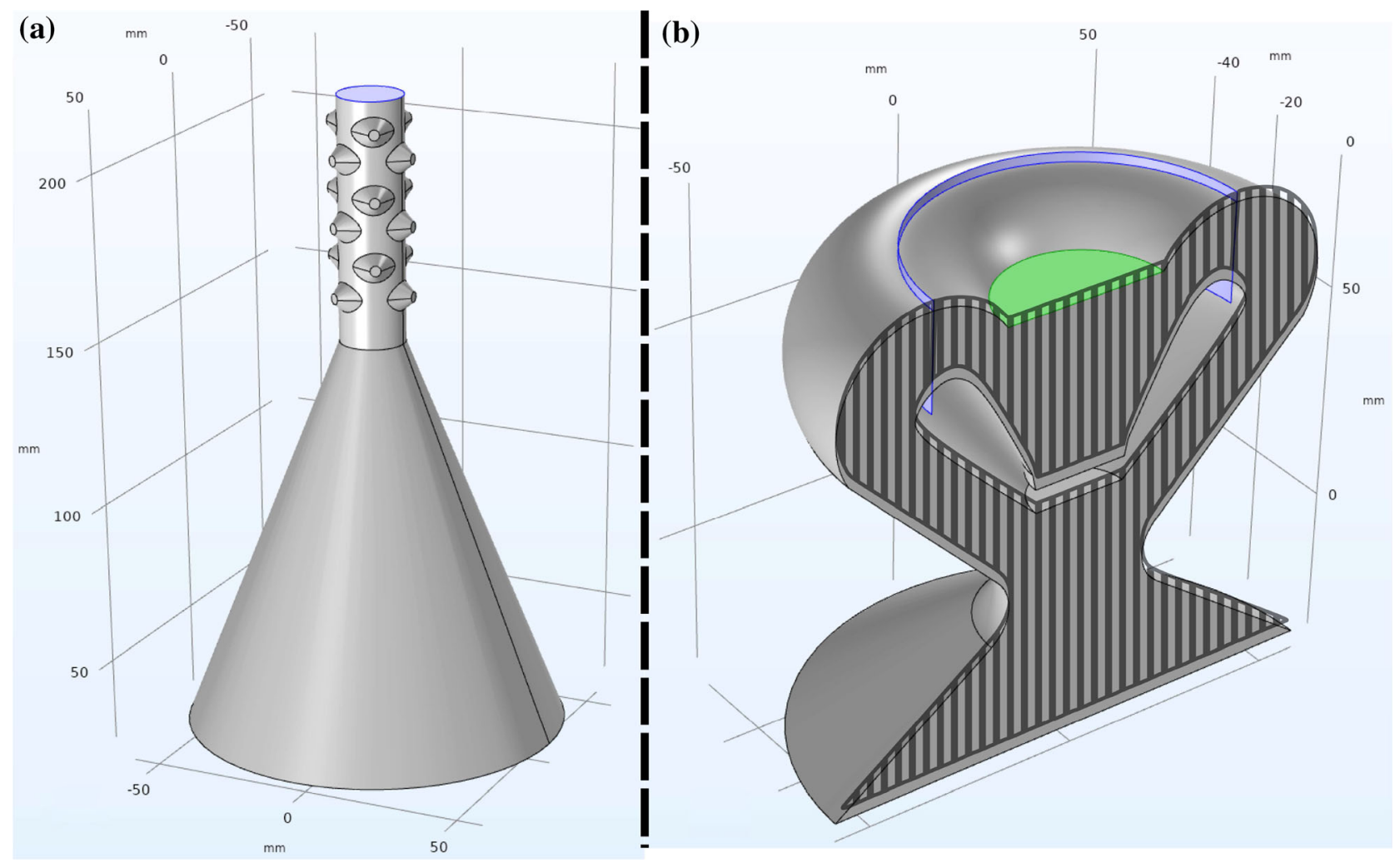

FIGURE 5. (a) Geometry corresponding to the boundary conditions imposed on the resolution of the flow for device one. In blue, we can see the surface type probe in which the flow rate has been calculated. (b) Geometry corresponding to the boundary conditions imposed on the resolution of the fluid dynamics in device two. In green, we can see the surface type probe in which the flow rate has been calculated. The surface type probe in blue color has been used to determine the minimum, maximum, and average normal speed to the surface in a steady state. The pattern of vertical lines surface is a symmetry boundary condition that reduces the computational domain in half

mate the mixing length or for regularization purposes. One way to compute the wall distance is to solve the eikonal equation $|\nabla D|=1$, with $D=0$ on solid walls and $\nabla D \cdot \hat{n}=0$ on other boundaries. COMSOL Multiphysics uses a modified eikonal equation based on the approach in ${ }^{8}$. This modification changes the dependent variable from $\mathrm{D}$ to $\mathrm{G}=1 / \mathrm{D}$. Equation $|\nabla D|=1$ then transforms to $\nabla G \cdot \nabla G=G^{4}$. Additionally, the modification adds some diffusion and multiplies $G^{4}$ by a factor to compensate for the diffusion. The result is the equation (3) where $\sigma_{w}$ is a small constant. If $\sigma_{w}$ is less than 0.5 , the maximum error falls off exponentially when $\sigma_{w}$ tends to zero. The default value of 0.2 is a good choice for both linear and quadratic elements.

To solve this system of coupled partial differential equations, several boundary conditions have been imposed. The walls have been imposed non-sliding: $\left.\mathbf{u}\right|_{\ell_{w}}=\overline{0}$. At the upper outlet of the filter, the pressure (relative to the atmospheric pressure) is free. That is, in the following equations,

$$
\hat{n}^{T}\left[-P \mathbf{I}+\left(\mu+\mu_{T}\right)\left(\nabla \cdot \mathbf{u}+(\nabla \cdot \mathbf{u})^{T}\right)\right] \hat{n}=-\hat{p}
$$

$$
\begin{gathered}
\hat{p} \geq p_{0} \\
\mathbf{u} \cdot \mathbf{t}=0 \\
\nabla G \cdot \hat{n}=0
\end{gathered}
$$

$p_{0}$ is the difference in pressure which is measured by the sensors. In summary, at the filter inlet, on the fan side, the boundary condition has also been the relative pressure measured by the BMP280 probes. Besides this, in both cases, normal flow to the surfaces is assumed. Figure 6(a) shows a velocity density map for various longitudinal cuts of the device.

In the second device the approach was analogous. However, the vorticity considerably lengthens the convergence (see Fig.7c)). It was for this reason that we used a symmetry plane, which is sketched with vertical pattern lines in Fig. 5(b), that reduced the size of the computational domain by half. As the calculations are scaled cubically with the size of the grid, this reduction facilitated and reduced the simulation time. The Symmetry boundary condition prescribes no penetra- 
(a)

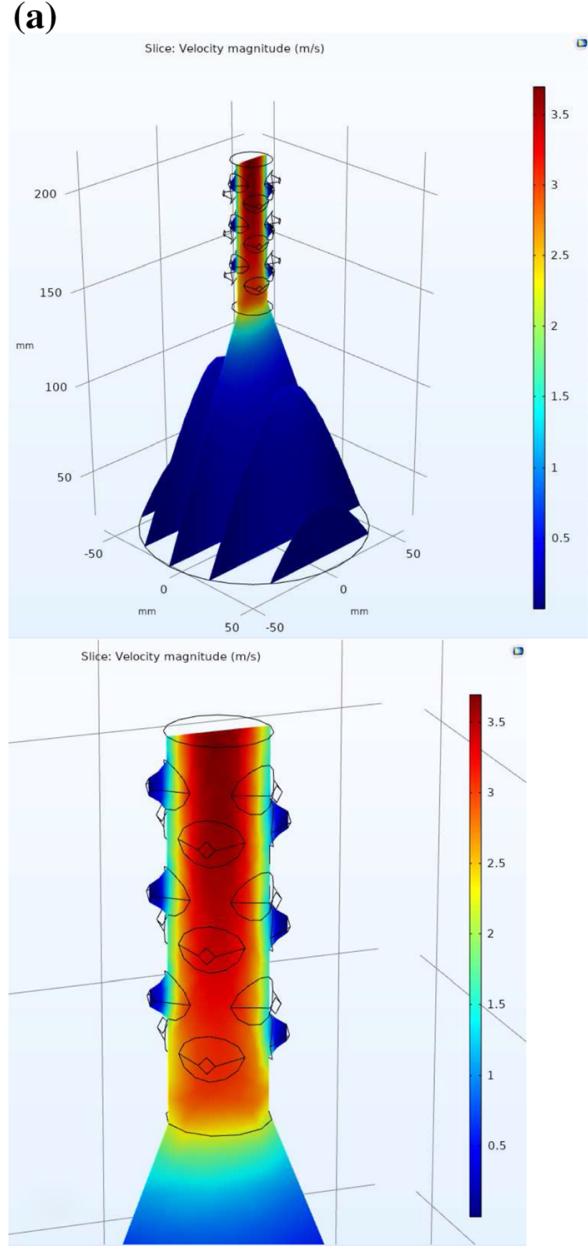

(b)

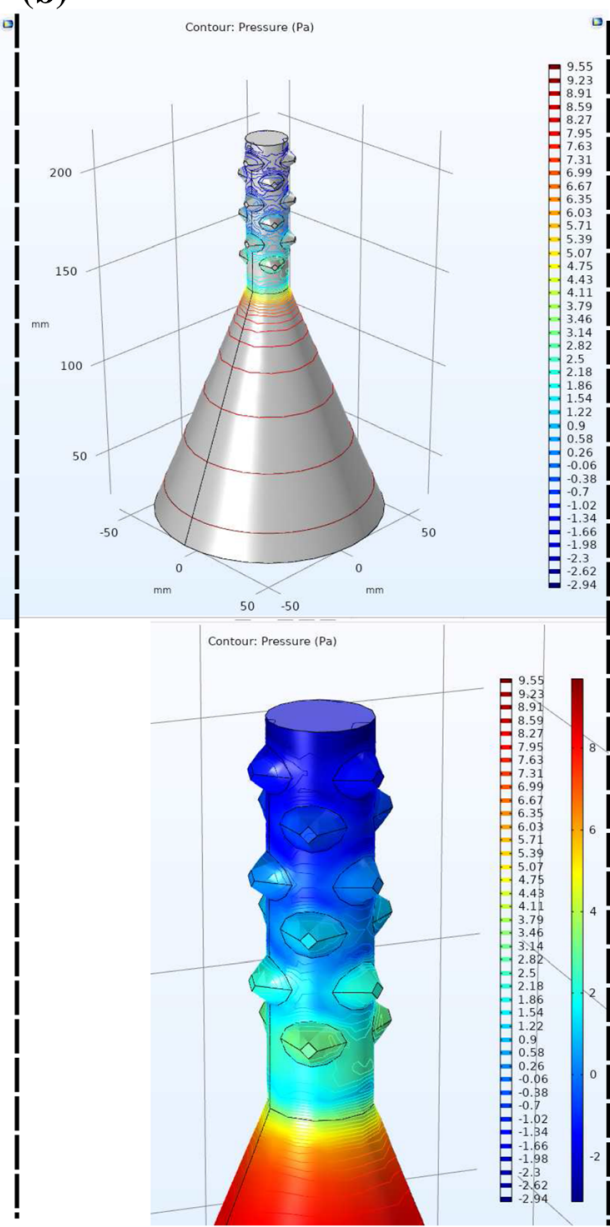

(c)

FIGURE 6. a) Speed density map in device one. A zoom of the speed map of the region illuminated by the UVC LEDs is also shown. b) Pressure density and pressure isoline map in device one. A zoom of the pressure map of the region illuminated by the UVC LEDs is also shown. c) Velocity field and streamlines

tion and vanishing shear stresses. The boundary condition is a combination of a Dirichlet condition and a Neumann condition for the compressible and incompressible formulations:

$\mathbf{u} \cdot \hat{n}=\overline{0}\left(-P \mathbf{I}+\left(\mu\left(\nabla \cdot \mathbf{u}+(\nabla \cdot \mathbf{u})^{T}\right)-\frac{2}{3} \mu(\nabla \cdot \mathbf{u})\right)\right)$
$\cdot \hat{n}=\overline{0}$

The Dirichlet condition takes precedence over the Neumann condition. The sense that this has is relative to the number of boundary conditions with respect to the partial derivative equations that we have. When the number of boundary conditions or constraints exceed the number of equations, the Dirichlet conditions will prevail over the Neumann ones. Therefore, the above equations are equivalent to the following equation for the incompressible formulation:

$$
\mathbf{u} \cdot \hat{n}=0 \mathbf{K}-(\mathbf{K} \cdot \hat{n}) \hat{n}=\overline{0}
$$

Where employing the strain rate tensor, we can define $\mathbf{K}$ as,

$$
\mathbf{K}=\mu\left(\nabla \cdot \mathbf{u}+(\nabla \cdot \mathbf{u})^{T}\right) \hat{n}
$$

Fig. 6(a) shows that, for device one working around $30 \%$ of its capacity, the average speed is around 3 meters per second and that it is higher in the cylinder axis, reaching more than 3.5 meters per second close to the outlet surface. In Fig. 5(a), we can see a surface probe that corresponds to the plane shown in blue. In this plane perpendicular to the air circulation, there is a flow rate of $0.084 \frac{L}{S}$. Figures $7(a)$ and $7(b)$ present the most relevant simulated results regarding fluid dynamics for the second device. In particular, we are interested in speeds/flow rate, and most especially, in the average speed in the region where the LEDs illuminate the flowing air. In Fig. 5(b), we can see a surface probe that corresponds to the plane shown in blue. In this plane perpendicular to the air current, the 

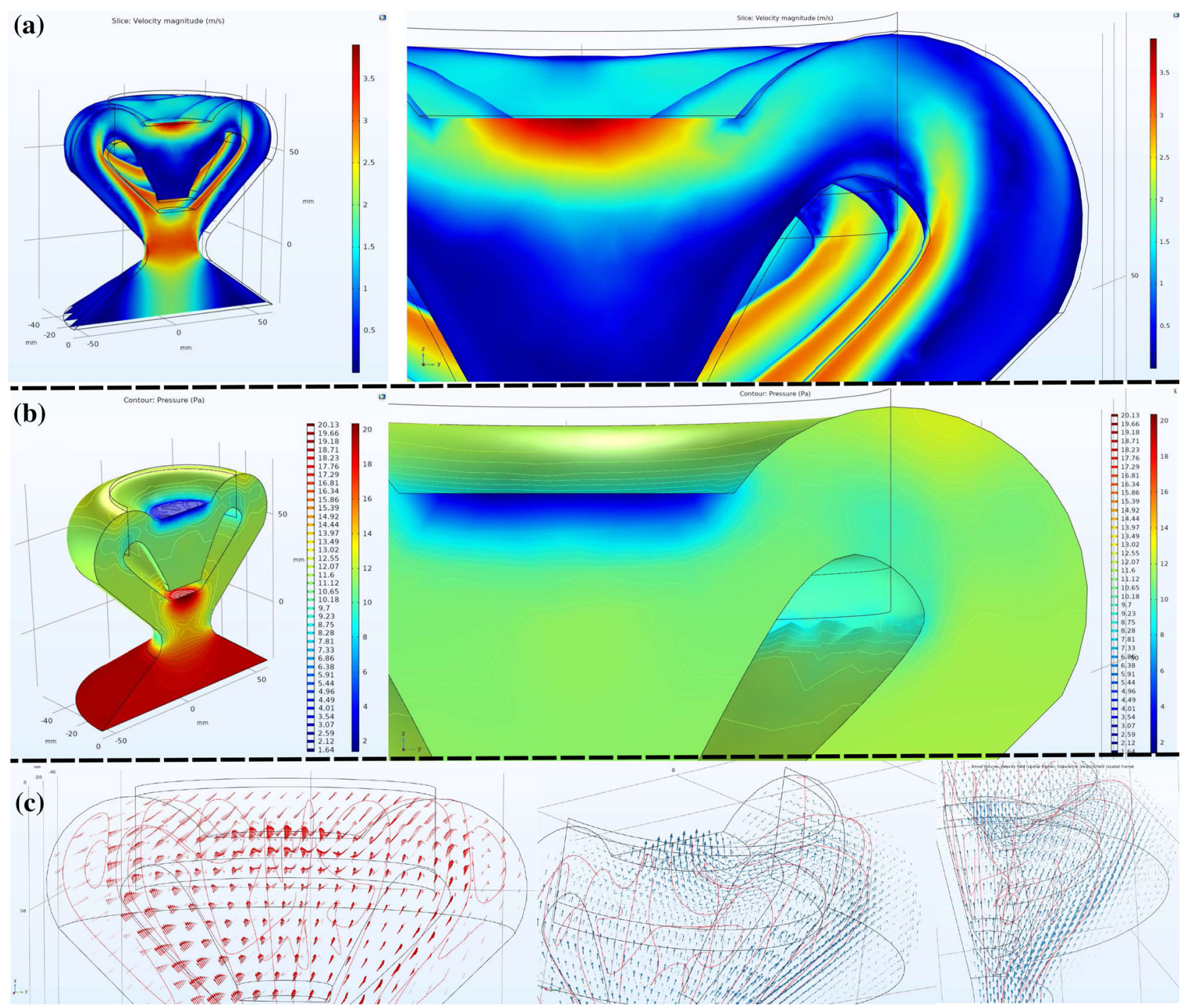

FIGURE 7. (a) Speed density map in device two. A zoom of the speed map of the region illuminated by the UVC LEDs is also shown. (b) Pressure density and pressure isoline map in device two. A zoom of the pressure map of the region illuminated by the UVC LEDs is also shown. (c) Velocity field and streamlines.

maximum value $\left(1.2 \frac{m}{s}\right)$, the minimum value $\left(0.3 \frac{m}{s}\right)$, and the average value $\left(0.42 \frac{m}{s}\right)$ of the fluid velocity that crosses it when the fan runs at $100 \%$ of its capacity are calculated. On average, the normal speed that crosses the plane probe is seven times slower than the one determined for the first device. In addition, we calculated the flow rate and velocity through the surface probe plotted in green color in Fig. 5(b). The result is $2.8 \frac{\mathrm{L}}{\mathrm{s}}$ flow rate and $2.5 \frac{\mathrm{m}}{\mathrm{s}}$ average velocity. Therefore we observe a flow rate more than thirty times the value obtained in device one.

\section{Radiation}

In ${ }^{17}$ we explain how to deal with the Helmholtz equation,

$$
\nabla \wedge \mu_{r}^{-1}(\nabla \wedge \mathbf{E})-k_{0}^{2}\left(\epsilon_{r}-\frac{j \sigma}{\omega \epsilon_{0}}\right) \mathbf{E}=\overline{0}
$$

in the frequency domain by using COMSOL Multiphysics. In COMSOL the package Frequency Domain Study is used to compute the response of a linear or linearized model subjected to harmonic excitation for one or several frequencies. In this particular case, we assume the diode frequency as the main harmonic frequency of the source. The source was introduced as a boundary condition which is called impedance boundary condition (IBC). This is a kind of absorbing boundary condition ${ }^{16}$ :

$$
\sqrt{\frac{\mu_{0} \mu_{r}}{\epsilon_{0} \epsilon_{r}-j \frac{\sigma}{\omega}}} \hat{n} \wedge \mathbf{H}+\mathbf{E}+\mathbf{E}_{i n c}=
$$




$$
\left(\hat{n} \cdot \mathbf{E}_{i n c}+\hat{n} \cdot \mathbf{E}\right)
$$

In ${ }^{17}$ we explain how to feed/illuminate a computational domain with this kind of source. We consider two kinds of boundary conditions that partially enclose the computational domain and fix a unique solution, the physical one. These are the perfect electric conductor (PEC) surface $\hat{n} \wedge \mathbf{E}=\overline{0}$ and the perfect magnetic conductor (PMC) surface $\hat{n} \wedge \mathbf{H}=\overline{0}$. All these boundary conditions are portrayed in Fig. 8(c). So as to reduce the computational domain, we study a simple diode in each geometry. Figure 8a) depicts the computational domain simulated for device one. In an identical form Fig. 8(b) illustrates the computational domain simulated for device two.

For device one, Fig. 9(a), on the left, shows the map of power density per unit area that is distributed in the filtering region. To the right and as a complement, we can see the electric field density. Similarly, in the
Fig. 9(b), on the left, illustrates for device two, the distribution of power per unit area. As before, we complement this figure with the one seen on the right, where we have the electric field density map.

In the next subsection, we draw some conclusions from these results, which combined with the flow rates, and therefore the exposure time give us the doses in units of energy per unit area.

\section{Doses}

For the first device, the exposure time is directly proportional to the number of rings of LEDs located in the cylindrical exit region and inversely proportional to the speed of the fluid. We could approach the problem by assuming that there is a continuous length of illumination and that this length of illumination is the radiated region. Then, the exposure time is proportional to that length, and therefore the dose re-

(c)

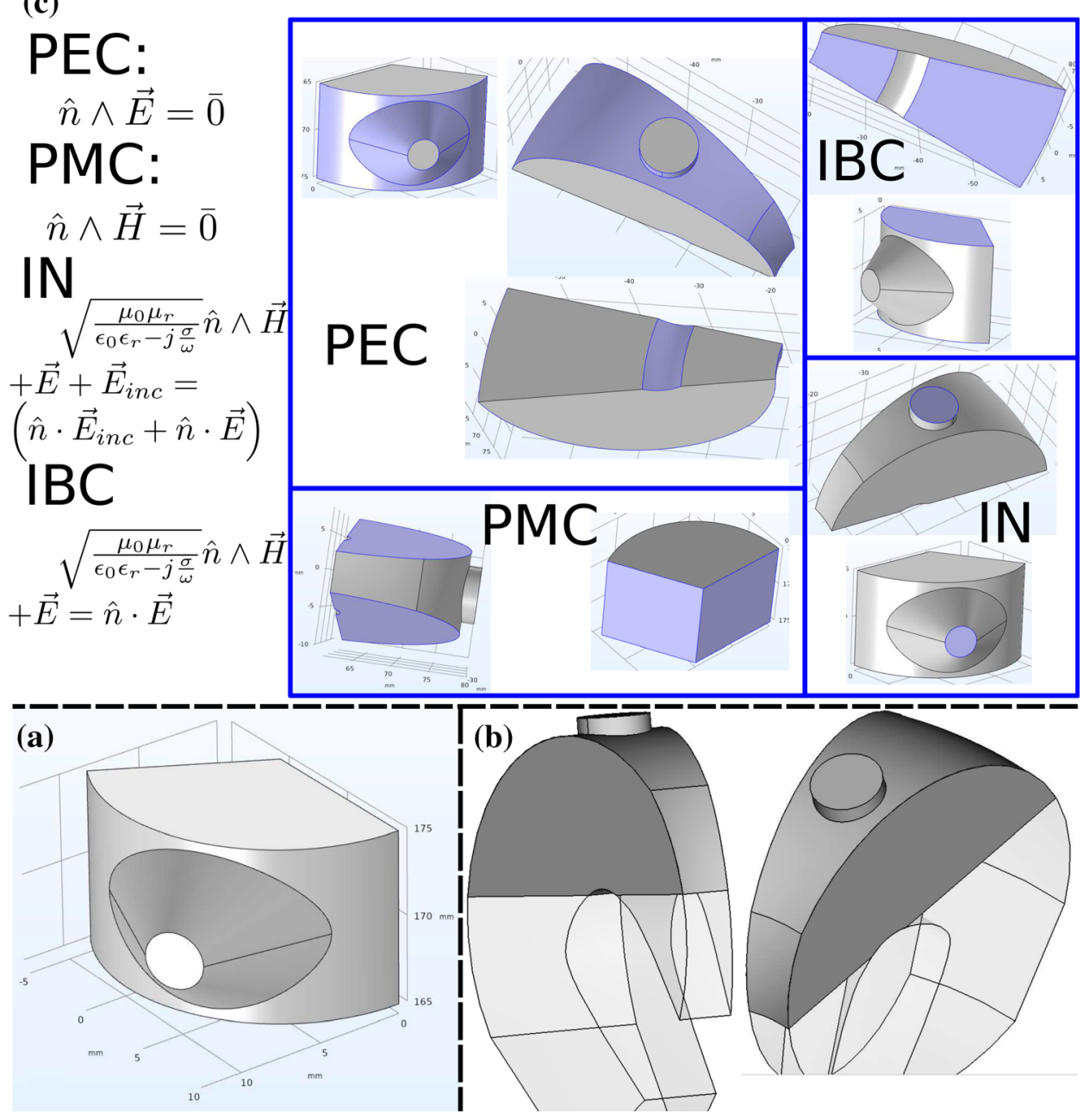

FIGURE 8. (a) The region where the electromagnetic simulation is performed for device one. (b) Region where the electromagnetic simulation is performed for device two. (c) Types and locations of the boundary conditions used in the resolution of the differential equation in partial derivatives of Helmholtz wave. 
(a)

(b)

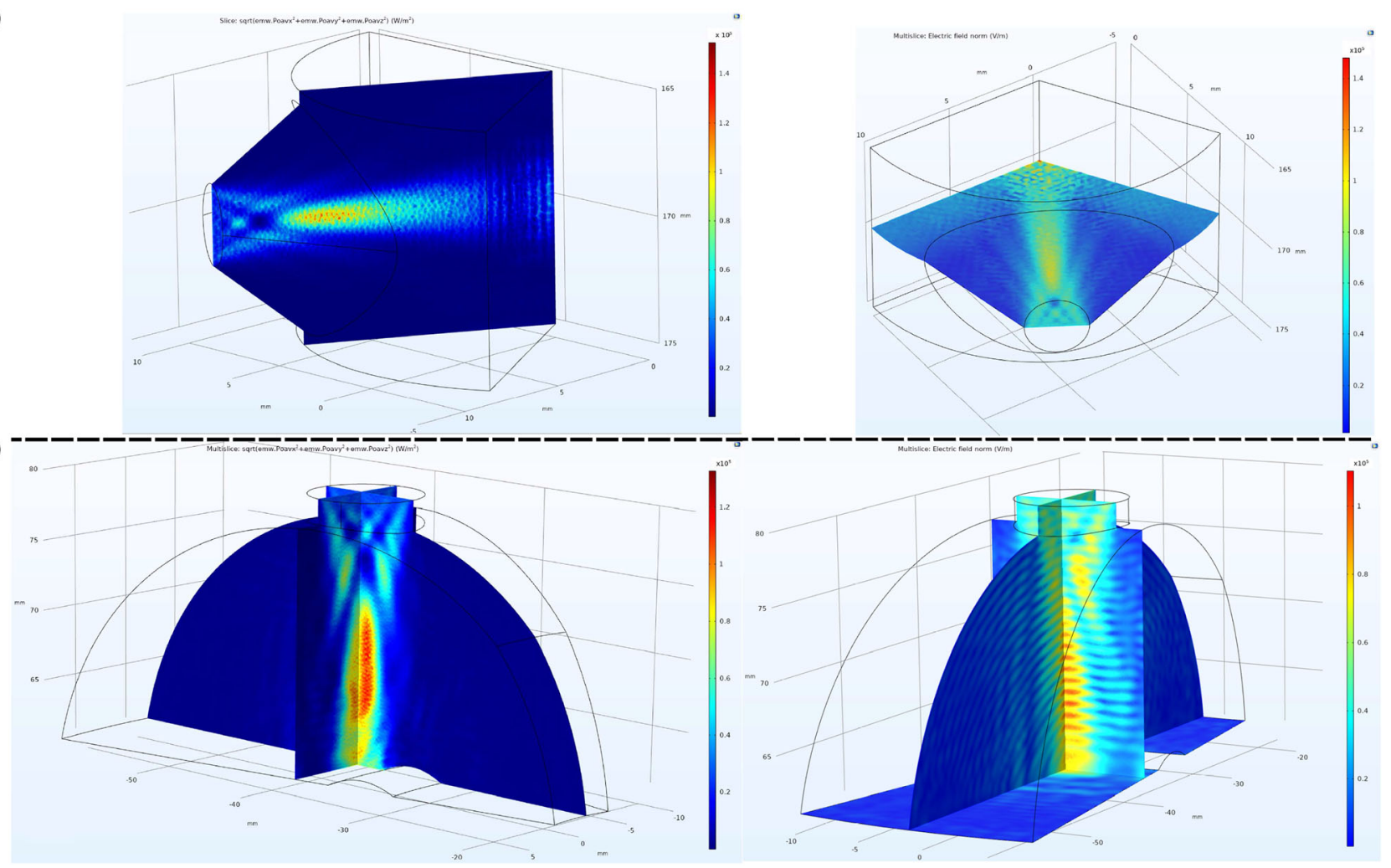

FIGURE 9. (a) On the left, the figure shows a two-dimensional density map or slice of the power per unit area for device one. On the right, the cut corresponding to the electric field density map is shown, also for device one. (b) On the left, the figure shows a two-dimensional density map or slice of the power per unit area for device two. On the right, the cut corresponding to the electric field density map is shown, also for device two.

ceived will be directly proportional to this radiated length, which is nothing other than the length of the irradiated cylinder. By considering a mean velocity of $3 \frac{\mathrm{m}}{\mathrm{s}}$ and an irradiated cylindrical length of $7.5 \mathrm{~cm}$, the exposure time for this device is $25 \mathrm{~ms}$. In Fig. 9(a) we can see a peak of irradiance around $1.5 \cdot 10^{5} \frac{\mathrm{W}}{\mathrm{m}^{2}}$. However, we should consider the average irradiance that is calculated in $4.5 \cdot 10^{4} \frac{\mathrm{W}}{\mathrm{m}^{2}}$. Hence, the fluence or doses in the filtering region for the first device is $112 \frac{\mathrm{mJ}}{\mathrm{cm}^{2}}$.

To determine the exposure time in the case of device two, the air in the illuminated region is assumed to have an exposure path of one centimeter, which implies an exposure time of $23.8 \mathrm{~ms}$ for an average speed of $0.42 \frac{\mathrm{m}}{\mathrm{s}}$. In Fig. 9(b) we can see this time a peak of irradiance around $1.3 \cdot 10^{5} \frac{\mathrm{W}}{\mathrm{m}^{2}}$. However, we should consider again the average irradiance that due to the volume under consideration is higher, $6.2 \cdot 10^{4} \frac{\mathrm{W}}{\mathrm{m}^{2}}$. Hence, the fluence or doses in the filtering region for the second device is $148 \frac{\mathrm{mJ}}{\mathrm{cm}^{2}}$.

\section{Construction details and measurements}

In the Fig. 3(a) we can see that device two has been printed in PLA with the appropriate geometry. Subsequently, the piece through which the air flows has been painted. It can be seen that red paint has been used. This painting is not merely decorative but serves two functions. The first and most important is to caulk, in the sense of waterproofing, the pores of the printed parts. This ensures that flow takes place inside the printed geometry and not through it. The second function is to shield and act as a resonance box for the lighting provided by the diodes. The paint that is basically roof waterproofing/sealing has been mixed with aluminum filings.

The air velocity has been measured with an anemometer https://www.pce-instruments.com/english/ api/getartfile?fnr $=1279276 \& \mathrm{dsp}=$ inlinePCE-009 which has a measurement range $[0.2,20.0] \pm 0.1 \frac{\mathrm{m}}{\mathrm{s}}$. The measured values, $2.9 \pm 0.1 \frac{\mathrm{m}}{\mathrm{s}}$ and $2.4 \pm 0.1 \frac{\mathrm{m}}{\mathrm{s}}$ for device one and two respectively, are extremely consistent with the simulations carried out. 

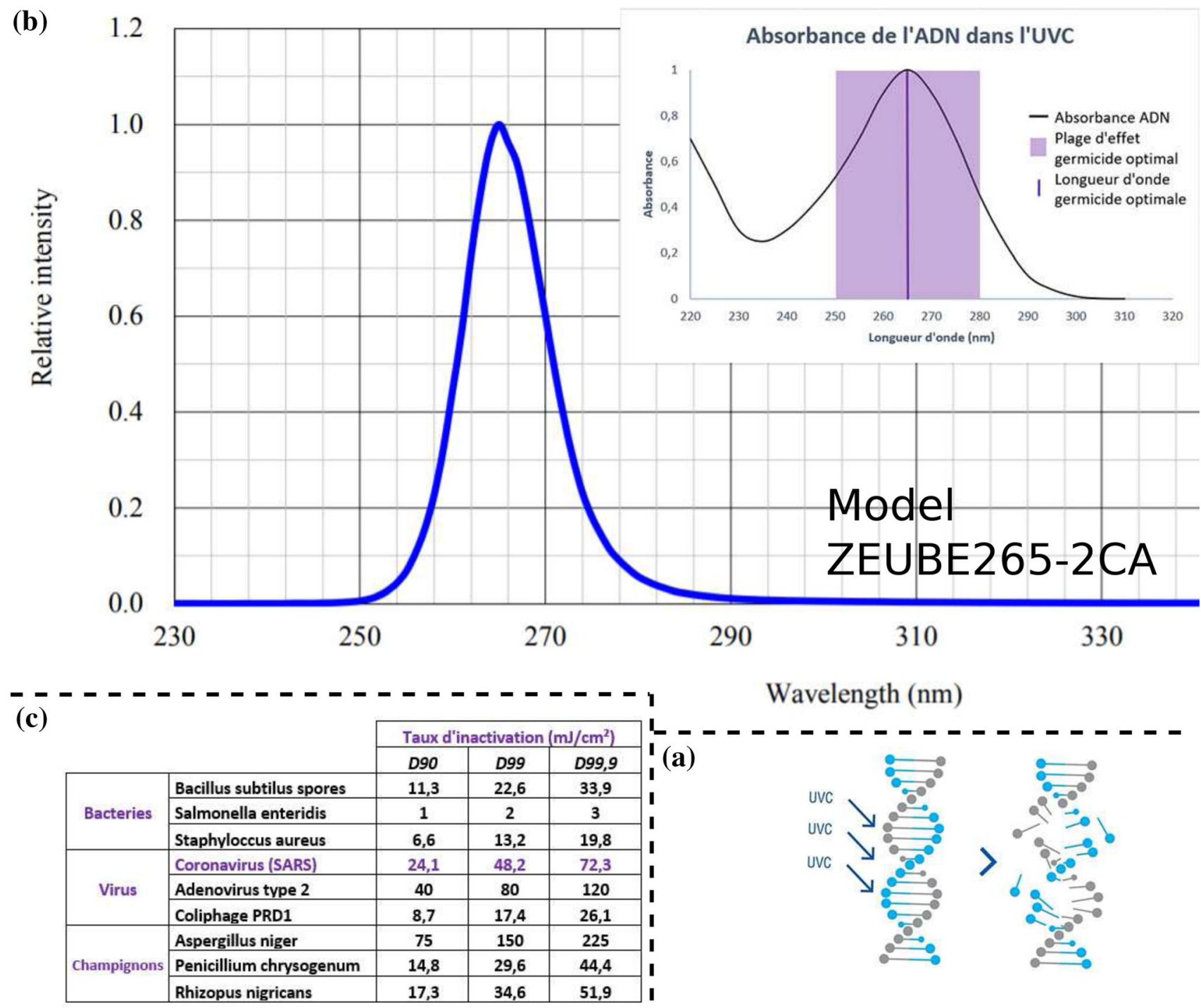

FIGURE 10. (a) The figure outlines the process by which type C ultraviolet radiation is capable of breaking down a DNA or RNA molecule and inactivating a pathogen. b) The illustration shows how the chosen diode has its emission maximum located at the maximum absorption peak of the DNA molecule. (c) The table synthesizes for some bacteria, viruses, and fungi the radioactive doses necessary to inactivate them with the safety of $90 \%, 99 \%$, and $99.9 \%$. the dose is established as a function of the energy per unit area that is applied to the regions where these pathogens are present

Combining the table in Fig. 10(c) and the radiation doses obtained in the previous section, we can infer that both devices are capable of deactivating pathogens with an efficiency greater than $99 \%$. In ${ }^{10}$ we can read:

"Results: The available data reveals large variations, which are apparently not caused by the coronaviruses but by the experimental conditions selected. If these are excluded as far as possible, it appears that coronaviruses are very UV sensitive. The upper limit determined for the log-reduction dose (90\% reduction) is approximately $10.6 \mathrm{~mJ} / \mathrm{cm}^{2}$ (median), while the true value is probably only $3.7 \mathrm{~mJ} / \mathrm{cm}^{2}$ (median).

\section{Conclusion}

Since coronaviruses do not differ structurally to any great exent, the SARS-CoV-2 virus -as well as possible future mutations- will very likely be highly UV sensitive, so that common UV disinfection procedures will inactivate the new SARS-CoV-2 virus without any further modification." From this sentence, we know that our criteria for coronaviruses, including the one responsible for COVID-19, deactivation provided by Fig. 10c) is realistic. Other sources as ${ }^{21,7}$ are in agreement with. ${ }^{10}$

However, as is clear from the summary in Table 1, device two is substantially better than one in all the valuations considered (including energy consumption). 
TABLE 1. Comparison between the devices performance.

( ${ }^{*}$ This prototype price should reduce its value in the market due to the massive production ${ }^{11}$ ).

\begin{tabular}{|c|c|c|c|c|}
\hline Devices & Flow rate $(\mathrm{L} / \mathrm{s})$ & Doses $\left(\mathrm{mJ} / \mathrm{cm}^{2}\right)$ & $\begin{array}{c}\text { Cost per } \\
\text { unit production* }\end{array}$ & $\begin{array}{l}\text { Simplicity of } \\
\text { construction }\end{array}$ \\
\hline I & 0.084 & 112 & Reference & $\begin{array}{l}\text { 3D printed parts: } 8 \\
\text { LEDs: } 18 \\
\text { Electronics }\end{array}$ \\
\hline II & 2.8 & 148 & $0.56 \%$ of Reference & $\begin{array}{l}\text { 3D printed parts: } 6 \\
\text { LEDs: } 10\end{array}$ \\
\hline
\end{tabular}

Device one has been designed to be able to deactivate pathogens, despite penalizing its filtering ratio in terms of flow. We conclude three things from this work. First of all, in a UVC filter, it is necessary to pay attention not only to the radiated power but to the dose that a pathogen would receive. In this sense, the devices must consider the radiation exposure time. The second conclusion is that complexity and electronics do not always provide the best performance. The third conclusion follows from the second and is that there is not or there is not always proportionality between the performance and the cost of a device. What is more, it is desirable that this relation does not exist. The reason, we would obtain the same service with fewer material resources and fewer work units.

\section{ACKNOWLEDGMENTS}

We would like to thank the support provided by La Fabrique de 1'industrie - Laboratoire d'idées in relation to the 3D-printers used in this work. Also, we would like to thank David Pallarés Aldeiturriaga which has supported partially one of the devices.

\section{REFERENCES}

${ }^{1}$ Kalapodas, D. I., and K. E. Paul. Monitor For UVC/IR Decontamination System, Patent Number: US 2011/ 0185302 A1, 2011. https://patents.google.com/patent/U S20150073396A1/en.

${ }^{2}$ About COMSOL 5.0. https://www.comsol.es/release/5.0.

${ }^{3}$ Al-Samkari, H., R. S. K. Leaf, W. H. Dzik, J. C. T. Carlson, A. E. Fogerty, A. Waheed, K. Goodarzi, P. K. Bendapudi, L. Bornikova, S. Gupta, D. E. Leaf, D. J. Kuter, and R. P. Rosovsky. COVID-19 and coagulation: bleeding and thrombotic manifestations of SARS-CoV-2 infection. Blood 136: 489-500, 2020.

${ }^{4}$ Amos, J., and C. Howard. How social distancing brought us closer as a BME community. Ann. Biomed. Eng. 48: 1443-1444, 2020.
${ }^{5}$ Batchelor, G. K. An Introduction to Fluid Dynamics. Press Syndicate of the University of Cambridge, 2000.

${ }^{6}$ Chen, Z., M. Zhong, L. Jiang, N. Chen, S. Tu, Y. Wei, L. Sang, X. Zheng, C. Zhang, J. Tao, L. Deng, and Y. Song. Effects of the lower airway secretions on airway opening pressures and suction pressures in critically Ill COVID-19 patients: a computational simulation. Ann. Biomed. Eng. 48: 3003-3013, 2020.

${ }^{7}$ Blatchley III, E. R., B. Petri, and W. Sun. SARS-CoV-2 UV Dose-Response Behavior. International Ultraviolet Association. https://iuva.org/.

${ }^{8}$ Fares, E., and W. Schröder. a differential equation for approximate wall distance. Int. J. Numer. Methods Fluids 39: 743-762, 2002.

${ }^{9}$ Filipovic, N., I. Saveljic, K. Hamada, and A. Tsuda. Abrupt deterioration of COVID-19 patients and spreading of SARS COV-2 virions in the lungs. Ann. Biomed. Eng. 48: 2705-2706, 2020.

${ }^{10}$ Heßling, M., K. Hönes, P. Vatter, and C. Lingenfelder. Ultraviolet irradiation doses for coronavirus inactivationreview and analysis of coronavirus photoinactivation studies. J. GMS Hyg Infect Control, 2020.

${ }^{11}$ How Did Mass Production Affect the Price of Consumer Goods? https://www.investopedia.com/ask/answers/05061 5/how-did-mass-production-affect-price-consumer-goods.a sp.

${ }^{12}$ Jones, J. P. Air Purification System, Patent Number: US 5925320, 1999. https://patents.google.com/patent/US5925320 A/en.

${ }^{13}$ Lewis, D. Why indoor spaces are still prime COVID hotspots. Nature 592: 22-25, 2021.

${ }^{14}$ Lichtblau, G. J. UVC air decontamination system, Patent Number: US 9327047 B1, 2016.

${ }^{15}$ Maloney, L. M., A. H. Yang, R. A. Princi, A. J. Eichert, D. R. Hébert, T. V. Kupec, A. E. Mertz, R. Vasyltsiv, T. M. Vijaya Kumar, G. J. Walker, E. J. Peralta, J. L. Hoffman, W. Yin, and C. R. Page. A COVID-19 airway management innovation with pragmatic efficacy evaluation: the patient particle containment chamber. Ann. Biomed. Eng. 48: 2371-2376, 2020.

${ }^{16}$ Moreno, E., Z. Hemmat, J. B. Roldán, M. F. Pantoja, A. R. Bretones, S. G. García, and R. Faez. Implementation of open boundary problems in photo-conductive antennas by using convolutional perfectly matched layers. IEEE Trans. Antennas Propag. 64(11): 4919-4922, 2016.

${ }^{17}$ Moreno, E., R. Sohrabi, G. Klochok, and E. Michael. Vertical versus planar pulsed photoconductive antennas that emit in the terahertz regime. Optik 166: 257-269, 2018.

${ }^{18}$ Radiation: Ultraviolet (UV) radiation. https://www.who. int/news-room/q-a-detail/radiation-ultraviolet-(uv). 
${ }^{19}$ Stanley, Data sheet of the part number : ZEUBE265-2CATR (https://www.stanley-components.com/php/download datafile.php?rp=0,ZEUBE265-2CA_e.pdf), Tech. rep., STANLEY ELECTRONIC CO., LTD, 2020.

${ }^{20}$ Terkelsen, J. Air sterilizer Unit, Patent Number: US 2021/ 0052764 A1, 2021.

${ }^{21}$ UVC FAQs https://www.goldenseauv.eu/fr/faq-fr/.

${ }^{22}$ Umar, M., F. Roddick, and L. Fan. Comparison of UVC lamp and UVC-light emitting diodes for treating municipal wastewater reverse osmosis concentrate. In: International Conference on Biological, Civil and Environmental Engineering (BCEE-2014) March 17-18, 2014, Dubai (UAE).

${ }^{23}$ What is Arduino? https://www.arduino.cc/en/Guide/Introd uction.
${ }^{24}$ Yuen, J. S.-K., K.-W. Cheung, L.-W. Tsoi, and K.-L. Lee, Ultraviolet air sterilising apparatus, Patent Number: GB 2405463 A, 2003.

${ }^{25}$ Zhang, K., and X. Liao. Theory and Modeling of Rotating Fluids. University Printing House, Cambridge CB2 8BS, United Kingdom, 2017.

${ }^{26}$ Zhengxiang, S., Z. Furong, L. Baoming, and C. Gang. Indoor air purification device, Patent Number: CN 104197425 (A) / CN 104197425 (B), 2014.

Publisher's Note Springer Nature remains neutral with regard to jurisdictional claims in published maps and institutional affiliations. 